\title{
The single nucleotide polymorphism upstream of insulin-induced gene 2 (INSIG2) is associated with the prevalence of hypercholesterolaemia, but not with obesity, in Japanese American women
}

\author{
Kenji Oki, Kiminori Yamane*, Nozomu Kamei, Takako Asao, Tomokazu Awaya and Nobuoki Kohno \\ Department of Molecular and Internal Medicine, Graduate School of Biomedical Sciences, Hiroshima University, 1-2-3 Kasumi, \\ Minami-ku, Hiroshima 734-8551, Japan
}

(Received 12 November 2007 - Revised 10 March 2008 - Accepted 8 April 2008 - First published online 23 June 2008)

Insulin-induced gene 2 (insig-2) protein is known to play important roles in cholesterol and TAG metabolism both in vivo and in vitro. One particularly interesting single nucleotide polymorphism (SNP), rs7566605, located $10 \mathrm{~kb}$ upstream of INSIG2 was reported to have the strongest association with obesity among 86604 SNP, while the relationship with dyslipidaemia is uncertain. Eight hundred and eighty-five Japanese Americans (347 men and 538 women) and 378 Japanese (182 men and 196 women) were enrolled, and the rs7566605 SNP, which is consistent with either G or C, was determined. We investigated the association between the rs7566605 SNP and the prevalence of hypercholesterolaemia or hypertriacylglycerolaemia, or obesity parameters, as assessed by BMI, waist girth and percentage body fat. There were no significant differences in BMI, waist girth and percentage body fat according to the genotype in each of the four groups, which was divided by population and sex. The prevalence of hypercholesterolaemia was significantly different between the genotypes in Japanese American female subjects (GG, $62.2 \%$; GC, $57.1 \%$; CC, $42.1 \% ; P=0.021$ ), but not in the other subjects. In Japanese American women, the subjects with the CC genotype had a 0.43 -fold decreased risk (95\% CI 0.24, 0.80) for hypercholesterolaemia compared with the GG genotype after adjustment for age, percentage body fat, smoking status and hormone replacement therapy. The CC genotype of the rs7566605 SNP is suggested to be a protective genetic factor against the progression of hypercholesterolaemia on a high-fat diet, especially in Japanese female subjects.

INSIG2: Polymorphisms: Dyslipidaemia: Obesity: High-fat diet

Insulin-induced gene insig proteins, including insig-1 and insig-2, play many important roles in regulating cholesterol or TAG synthesis, mainly in the liver. 3-Hydroxy-3-methylglutaryl (HMG)-CoA reductase, a rate-limiting enzyme in cholesterol synthesis, catalyses the reduction of HMG-CoA to mevalonate ${ }^{(1)}$. Overexpression of insig proteins can activate the degradation of HMG-CoA reductase in Chinese hamster ovary cells in vitro ${ }^{(2)}$. The knockdown of insig-2 by RNA interference suppresses the degradation of HMG-CoA reductase in SV-589 cells ${ }^{(3)}$. In addition, insig-2-overexpressing mice exhibit lower plasma TAG levels compared with wild-type mice, independent of food intake, body weight and fat weight ${ }^{(4)}$, which suggests the association between insig-2 and TAG metabolism. Taken together, it is suggested that insig-2 is strongly related to cholesterol and TAG metabolism through the level of insig-2 expression.

Recently, an interesting single nucleotide polymorphism (SNP), rs7566605, which is located $10 \mathrm{~kb}$ upstream of INSIG2, was found to be associated with obesity, as assessed by $\mathrm{BMI}^{(5)}$. This polymorphism was identified from a genomewide association scan of 86604 SNP in the Framingham Heart Study offspring cohort. In the report, the relationship with obesity was confirmed in four out of five additional cohorts ${ }^{(5)}$. Moreover, Lyon et al. identified a significant association between the SNP and obesity in five large cohorts, but detected no correlation in three other cohorts ${ }^{(6)}$. The relationship between the SNP and the degree of obesity is extremely interesting, because it was suggested that the key factors to resolving the controversy would be the degree of BMI. In short, the SNP is likely to influence BMI in obese subjects rather than lean subjects ${ }^{(5,6)}$.

We hypothesised that the rs7566605 SNP is associated with hypercholesterolaemia or hypertriacylglycerolaemia, since the polymorphism, which is located upstream of INSIG2, may influence the expression of insig-2. Therefore, we investigated the association between the SNP and the prevalence of hypercholesterolaemia or hypertriacylglycerolaemia as well as obesity in Japanese American and Japanese subjects.

In this Japanese American population-based study, the subjects have been reported to develop dyslipidaemia and obesity more rapidly than Japanese living in Japan because of a Westernised food habit in which Japanese Americans ingested more fat of animal origin and of a lower PUFA:SFA ratio than native Japanese $^{(7,8)}$. Therefore, we considered that these populations

Abbreviations: \%fat, percentage body fat; HMG, 3-hydroxy-3-methylglutaryl; HOMA-IR, homeostasis model assessment for insulin resistance; SNP, single nucleotide polymorphism.

* Corresponding author: Dr Kiminori Yamane, fax +81 82255 7360, email yamanek@hiroshima-u.ac.jp 
were suitable for resolving the association between the SNP and dyslipidaemia or obesity, because any genetic susceptibility influenced by differences of food habits would be estimated in comparing between Japanese Americans and Japanese.

\section{Subjects and methods}

\section{Study population}

We enrolled Japanese Americans in medical surveys in Hawaii and Los Angeles, California, and Japanese living in Japan. The survey, which is called the Hawaii-Los Angeles-Hiroshima study, has continued since 1970 and has been described elsewhere in detail ${ }^{(7)}$. In brief, the survey represented a long-term epidemiological study of the risk factors for dyslipidaemia, obesity, diabetes, hypertension and atherosclerosis. The subjects living in Hawaii or Los Angeles were limited to a population who originated genetically from the Japanese.

The study subjects were Japanese Americans enrolled in a medical survey in 2002 and 2004, and Japanese living in Japan who had undergone similar medical examinations in 2004. We analysed 885 Japanese Americans (347 men with a mean age of 64.9 (SD 14.8) years and 538 women with a mean age of 65.6 (SD 13.8) years) and 378 Japanese (182 men with a mean age of 52.4 (SD 13.3) years and 196 women with a mean age of 58.2 (SD 10.4) years). All subjects provided written informed consent. The present study was approved by the ethics committee of Hiroshima University and the Council of the Hiroshima Kenjin-Kai Association in Hawaii and Los Angeles.

\section{Measurements}

The height, body weight and waist girth of the subjects were measured using standard methods, and the BMI was calculated. The percentage body fat (\%fat) was assessed by measuring the bioelectric impedance from foot to foot ${ }^{(9)}$. The smoking status (current, past, or none) was assessed using standard interviewing procedures. Venous blood was obtained in the morning after overnight fasting. The blood samples were centrifuged, and the obtained serum was immediately frozen. Frozen samples were brought back to Japan and analysed together with the samples obtained in Japan in the same laboratory. Serum total cholesterol and TAG levels were measured using an enzymic method with a 736-60E Auto Analyser (Hitachi, Tokyo, Japan $)^{(10)}$. HDL-cholesterol levels were determined using a precipitation method ${ }^{(11)}$. Serum glucose levels were measured by the glucose oxidase method. Serum concentrations of insulin were measured by a double-antibody RIA. Dyslipidaemia was diagnosed as HDL-cholesterol $<400 \mathrm{mg} / \mathrm{l}$, total cholesterol $\geq 2200 \mathrm{mg} / \mathrm{l}, \mathrm{TAG} \geq 1500 \mathrm{mg} / \mathrm{l}$, or in patients receiving antidyslipidaemic medicine, such as statins, cholestyramine, fibrate, or others. Hypercholesterolaemia was diagnosed as total cholesterol $\geq 2200 \mathrm{mg} / \mathrm{l}$ or in patients receiving statins or cholestyramine, and hypertriacylglycerolaemia was diagnosed as TAG $\geq 1500 \mathrm{mg} / \mathrm{l}$ or in patients receiving fibrate. In Japanese subjects, the details of antidyslipidaemic medication had been undetermined. Thus, the subjects with antidyslipidaemic medications were excluded in considering the prevalence of hypercholesterolaemia or hypertriacylglycerolaemia in Japanese men and women. The homeostasis model assessment for insulin resistance
(HOMA-IR) was calculated as follows: fasting serum insulin $(\mathrm{pmol} / \mathrm{l}) \times$ fasting serum glucose $(\mathrm{mmol} / \mathrm{l}) / 2 \cdot 5^{(12)}$.

Genomic DNA was extracted from peripheral leucocytes. The rs7566605 SNP, which is consistent with either G or C, was determined by the ABI fluorescence-based assay-bydesign allelic discrimination method (Applied Biosystems, Foster City, CA, USA). Accuracy of genotyping using the method was validated in some cases by comparing the results with sequence data obtained by a PCR-based direct sequencing method.

\section{Statistical analysis}

Quantitative data are presented as the mean values and standard deviations for continuous normally distributed variables. During analysis, the values for serum insulin levels and HOMA-IR were converted into their $\log$ values and are expressed as medians and interquartile ranges, because the distribution of the data is skewed. Analyses were performed using SPSS for Windows (release 12.0; SPSS Inc., Chicago, IL, USA). $P$ values $<0.05$ were considered to be significant. Allele and genotype frequencies were analysed using the gene-counting method and the Hardy-Weinberg equilibrium, and confirmed by the $\chi^{2}$ test.

All analyses were performed in both Japanese Americans and Japanese males and females. First, to compare BMI, waist girth and \%fat according to the genotype, statistical significance was determined by one-way ANOVA. When we examined the association between the SNP and serum insulin levels or HOMA-IR, subjects with a history of diabetes, receiving treatment for diabetes, or serum fasting glucose levels higher than $1600 \mathrm{mg} / \mathrm{l}$ were excluded. Power analyses to detect the association between BMI and the SNP were performed to confirm the sufficient sample size in each of the four groups, as reference to the study reported by Herbert et al. ${ }^{(5)}$. Second, the associations between the SNP and the prevalence of dyslipidaemia, hypercholesterolaemia, or hypertriacylglycerolaemia were analysed by the $\chi^{2}$ test. Furthermore, the same analyses were performed after the Japanese American women were divided into those with the $\mathrm{G}$ allele and $\mathrm{CC}$ genotype or the GG genotype and $\mathrm{C}$ allele. Third, OR, corresponding $95 \% \mathrm{CI}$, and standard coefficient for the prevalence of hypercholesterolaemia were determined by logistic regression analyses taking into the SNP, age, \%fat, smoking status and hormone replacement therapy in Japanese American female subjects.

\section{Results}

In Japanese Americans, the allele frequency of the rs7566605 SNP was 0.679 for $\mathrm{G}$ and 0.321 for $\mathrm{C}$, and the genotype distribution was as follows: GG, $46.6 \%$; GC, $42.6 \%$; CC, $10.8 \%$. In Japanese, the allele frequency was 0.642 for $\mathrm{G}$ and 0.358 for $\mathrm{C}$, and the distribution (GG, 39.4\%; GC, 49.5\%; CC, $11.1 \%$ ) assessed by the $\chi^{2}$ test did not differ from that in Japanese Americans. Genotypes did not deviate significantly from the expected Hardy-Weinberg frequencies.

The subjects were divided into four groups according to population and sex, and the subjects' clinical and biological characteristics according to the genotypes are shown in Tables 1 and 2 . In each of the four groups, there were no significant 
Table 1. Clinical and biological characteristics in Japanese American or Japanese men

(Mean values and standard deviations, median values and interquartile ranges, or frequencies)

\begin{tabular}{|c|c|c|c|c|c|c|c|c|c|c|c|c|c|c|}
\hline & \multicolumn{7}{|c|}{ Japanese American men } & \multicolumn{7}{|c|}{ Japanese men } \\
\hline & \multicolumn{2}{|c|}{$\mathrm{GG}(n 171)$} & \multicolumn{2}{|c|}{$\mathrm{GC}(n 137)$} & \multicolumn{2}{|c|}{$\mathrm{CC}(n 39)$} & \multirow[b]{2}{*}{$P$} & \multicolumn{2}{|c|}{$\mathrm{GG}(n 68)$} & \multicolumn{2}{|c|}{$\mathrm{GC}(n 94)$} & \multicolumn{2}{|c|}{ CC (n 20) } & \multirow[b]{2}{*}{$P$} \\
\hline & Mean & SD & Mean & SD & Mean & SD & & Mean & SD & Mean & SD & Mean & SD & \\
\hline Age (years) & $65 \cdot 0$ & $15 \cdot 0$ & $64 \cdot 3$ & 14.4 & 66.5 & $15 \cdot 3$ & 0.697 & $51 \cdot 0$ & 11.9 & $51 \cdot 0$ & $14 \cdot 3$ & $55 \cdot 1$ & $13 \cdot 3$ & 0.431 \\
\hline BMI $\left(\mathrm{kg} / \mathrm{m}^{2}\right)$ & 24.8 & 3.5 & $25 \cdot 4$ & 4.0 & 24.7 & 3.4 & 0.352 & $24 \cdot 0$ & $3 \cdot 2$ & 23.6 & $3 \cdot 1$ & 24.4 & $2 \cdot 8$ & 0.546 \\
\hline Waist girth (cm) & 87.7 & 9.9 & 88.8 & 9.9 & 87.7 & 8.6 & 0.618 & $82 \cdot 3$ & 8.4 & 81.8 & 8.2 & 84.8 & $7 \cdot 7$ & 0.337 \\
\hline Body fat $(\%)$ & $24 \cdot 2$ & $6 \cdot 2$ & $25 \cdot 3$ & $6 \cdot 2$ & $24 \cdot 0$ & $6 \cdot 3$ & 0.280 & 23.4 & $5 \cdot 8$ & $22 \cdot 1$ & 4.7 & $24 \cdot 0$ & 4.8 & 0.150 \\
\hline Total cholesterol (mg/l) & 2067 & 372 & 2071 & 374 & 2137 & 446 & 0.573 & 2057 & 279 & 2047 & 344 & 2151 & 339 & 0.417 \\
\hline \multicolumn{15}{|l|}{ TAG $(\mathrm{mg} / \mathrm{l})$} \\
\hline Median & \multirow{2}{*}{\multicolumn{2}{|c|}{$\begin{array}{c}1260 \\
825-1873\end{array}$}} & \multirow{2}{*}{\multicolumn{2}{|c|}{$\begin{array}{c}1350 \\
875-2210\end{array}$}} & \multirow{2}{*}{\multicolumn{2}{|c|}{$\begin{array}{c}1120 \\
910-1800\end{array}$}} & 0.113 & \multirow{2}{*}{\multicolumn{2}{|c|}{$\begin{array}{c}1265 \\
845-1855\end{array}$}} & \multirow{2}{*}{\multicolumn{2}{|c|}{$\begin{array}{c}1080 \\
820-1468\end{array}$}} & \multirow{2}{*}{\multicolumn{2}{|c|}{$\begin{array}{c}1225 \\
630-1918\end{array}$}} & 0.250 \\
\hline Interquartile range & & & & & & & & & & & & & & \\
\hline HDL-cholesterol (mg/l) & 523 & 142 & 501 & 122 & 493 & 114 & 0.237 & 559 & 156 & 537 & 117 & 543 & 111 & 0.579 \\
\hline \multicolumn{15}{|l|}{ Dyslipidaemia therapy } \\
\hline$n$ & \multicolumn{2}{|c|}{33} & \multicolumn{2}{|c|}{21} & \multicolumn{2}{|c|}{4} & 0.337 & \multicolumn{2}{|c|}{4} & \multicolumn{2}{|c|}{3} & \multicolumn{2}{|c|}{0} & 0.434 \\
\hline$\%$ & \multirow{2}{*}{\multicolumn{2}{|c|}{$\begin{array}{l}19 \cdot 3 \\
30\end{array}$}} & & & & & & & & & & & & \\
\hline Statins or cholestyramine $(n)$ & & & & & & & & & & & & & & \\
\hline Fibrate $(n)$ & & & & & & & & & & & & & & \\
\hline Others $(n)$ & & & & & & & & & & & & & & \\
\hline Dyslipidaemia & & & & & & & & & & & & & & \\
\hline$n$ & & & & & & & 0.335 & & & & & & & 0.121 \\
\hline$\%$ & & & & & & & & & & & & & & \\
\hline Hypercholesterolaemia & & & & & & & & & & & & & & \\
\hline$n$ & & & & & & & 0.872 & & & & & & & 0.068 \\
\hline$\%$ & & & & & & & & & & & & & & \\
\hline Hypertriacylglycerolaemia & & & & & & & & & & & & & & \\
\hline n & & & & & & & 0.583 & & & & & & & 0.232 \\
\hline$\%$ & & & & & & & & & & & & & & \\
\hline Serum insulin $(\mu \mathrm{U} / \mathrm{ml})$ & & & & & & & & & & & & & & \\
\hline Median & & & & & & & 0.785 & & & & & & & 0.629 \\
\hline Interquartile range & & & & & & & & & & & & & & \\
\hline HOMA-IR & & & & & & & & & & & & & & \\
\hline Median & & & & & & & 0.937 & & & & & & & 0.567 \\
\hline Interquartile range & 0.91 & 16 & 0.8 & 44 & 1.0 & .04 & & 0.83 & .97 & $0.7 \xi$ & .76 & 0.65 & 41 & \\
\hline
\end{tabular}

HOMA-IR, homeostasis model assessment for insulin resistance.
${ }^{*}$ Statistical significance was determined by one-way ANOVA. 
Table 2. Clinical and biological characteristics in Japanese American or Japanese women*

(Mean values and standard deviations, median values and interquartile ranges, or frequencies)

\begin{tabular}{|c|c|c|c|c|c|c|c|c|c|c|c|c|c|c|}
\hline & \multicolumn{7}{|c|}{ Japanese American women } & \multicolumn{7}{|c|}{ Japanese women } \\
\hline & \multicolumn{2}{|c|}{$\mathrm{GG}(n 241)$} & \multicolumn{2}{|c|}{ GC $(n 240)$} & \multicolumn{2}{|c|}{$\mathrm{CC}(n 57)$} & \multirow[b]{2}{*}{$P$} & \multicolumn{2}{|c|}{$\mathrm{GG}(n 81)$} & \multicolumn{2}{|c|}{ GC ( $(n 93)$} & \multicolumn{2}{|c|}{ CC (n 22) } & \multirow[b]{2}{*}{$P$} \\
\hline & Mean & SD & Mean & SD & Mean & SD & & Mean & SD & Mean & SD & Mean & SD & \\
\hline Age (years) & $65 \cdot 4$ & $14 \cdot 3$ & $66 \cdot 0$ & $13 \cdot 2$ & 64.5 & $14 \cdot 7$ & 0.734 & 57.5 & $11 \cdot 3$ & 58.5 & $9 \cdot 2$ & $59 \cdot 4$ & 11.8 & 0.705 \\
\hline BMI $\left(\mathrm{kg} / \mathrm{m}^{2}\right)$ & $23 \cdot 2$ & 3.8 & $23 \cdot 1$ & 3.7 & $23 \cdot 0$ & $4 \cdot 1$ & 0.912 & 23.0 & $2 \cdot 6$ & $23 \cdot 2$ & 3.0 & $23 \cdot 2$ & 3.3 & 0.859 \\
\hline Waist girth $(\mathrm{cm})$ & 76.9 & 8.8 & 76.9 & $9 \cdot 3$ & $76 \cdot 5$ & $11 \cdot 3$ & 0.966 & $77 \cdot 1$ & 8.3 & 78.7 & 9.0 & $78 \cdot 7$ & 9.4 & 0.429 \\
\hline Percentage body fat (\%) & $31 \cdot 2$ & $7 \cdot 6$ & $30 \cdot 8$ & $7 \cdot 1$ & 30.9 & 8.0 & 0.880 & $29 \cdot 1$ & 5.0 & $29 \cdot 1$ & 6.0 & $29 \cdot 7$ & 6.9 & 0.890 \\
\hline Total cholesterol (mg/l) & 2200 & 389 & 2193 & 389 & 2213 & 474 & 0.939 & 2181 & 357 & 2202 & 309 & 2150 & 354 & 0.787 \\
\hline \multicolumn{15}{|l|}{ TAG $(\mathrm{mg} / \mathrm{l})$} \\
\hline Median & \multirow{2}{*}{\multicolumn{2}{|c|}{$\begin{array}{c}1170 \\
795-1785\end{array}$}} & \multirow{2}{*}{\multicolumn{2}{|c|}{$\begin{array}{c}1140 \\
830-1668\end{array}$}} & \multirow{2}{*}{\multicolumn{2}{|c|}{$\begin{array}{c}1080 \\
860-1695\end{array}$}} & \multirow[t]{2}{*}{0.897} & \multirow{2}{*}{\multicolumn{2}{|c|}{$\begin{array}{c}770 \\
630-1240\end{array}$}} & \multirow{2}{*}{\multicolumn{2}{|c|}{$\begin{array}{c}810 \\
630-1040\end{array}$}} & \multirow{2}{*}{\multicolumn{2}{|c|}{$\begin{array}{c}730 \\
600-1170\end{array}$}} & 0.569 \\
\hline Interquartile range & & & & & & & & & & & & & & \\
\hline HDL-cholesterol (mg/l) & 603 & 136 & 594 & 149 & 587 & 139 & 0.637 & 612 & 112 & 631 & 126 & 628 & 128 & 0.575 \\
\hline Dyslipidaemia therapy & & & & & & & & & & & & & & \\
\hline$n$ & \multicolumn{2}{|c|}{45} & \multicolumn{2}{|c|}{45} & \multicolumn{2}{|c|}{6} & 0.312 & \multicolumn{2}{|c|}{8} & \multicolumn{2}{|c|}{11} & \multicolumn{2}{|c|}{0} & 0.244 \\
\hline$\%$ & \multirow{2}{*}{\multicolumn{2}{|c|}{$\begin{array}{c}18 \cdot 7 \\
40\end{array}$}} & & & & & & & & & & & & \\
\hline Statins or cholestyramine $(n)$ & & & & & & & & & & & & & & \\
\hline Fibrate $(n)$ & & & & & & & & & & & & & & \\
\hline Others $(n)$ & & & & & & & & & & & & & & \\
\hline Dyslipidemia & & & & & & & & & & & & & & \\
\hline$n$ & & & & & & & 0.009 & & & & & & & 0.918 \\
\hline$\%$ & & & & & & & & & & & & & & \\
\hline Hypercholesterolaemia & & & & & & & & & & & & & & \\
\hline$n$ & & & & & & & 0.021 & & & & & & & 0.930 \\
\hline$\%$ & & & & & & & & & & & & & & \\
\hline Hypertriacylglycerolaemia & & & & & & & & & & & & & & \\
\hline$n$ & & & & & & & 0.701 & & & & & & & 0.549 \\
\hline$\%$ & & & & & & & & & & & & & & \\
\hline Serum insulin $(\mu \mathrm{U} / \mathrm{ml})$ & & & & & & & & & & & & & & \\
\hline Median & & & & & & & 0.878 & & & & & & & 0.686 \\
\hline Interquartile range & & & & & & & & & & & & & & \\
\hline HOMA-IR & & & & & & & & & & & & & & \\
\hline Median & & & & & & & 0.884 & & & & & & & 0.718 \\
\hline Interquartile range & $0.7 \mathrm{~s}$ & .93 & 0.7 & .89 & 0.7 & & & 0.5 & & 0.6 & 17 & 0.6 & .67 & \\
\hline
\end{tabular}

HOMA-IR, homeostasis model assessment for insulin resistance.

Statistical significance was determined by one-way ANOVA. 
differences in age, BMI, waist girth, \%fat, serum insulin levels and HOMA-IR among genotypes. In both Japanese American men and women, the power to detect the association between BMI and the SNP was more than $90 \%$. In Japanese men and women, the power was more than $80 \%$. In both Japanese American and Japanese men, there was no relationship between the SNP and the prevalence of dyslipidaemia, hypercholesterolaemia or hypertriacylglycerolaemia (Table 1). The prevalence of dyslipidaemia was significantly different between the genotypes in Japanese American women (GG, 73.4\%; GC, 69.2\%; CC, $52.6 \% ; P=0.009$ ), but not in Japanese women (Table 2). In particularly, a significant association was obtained in the prevalence of hypercholesterolaemia (GG, $62.2 \%$; GC, $57.1 \%$; CC, $42.1 \%$; $P=0.021$ ), but not in that of hypertriacylglycerolaemia (GG, $32.0 \%$; GC, $33.8 \%$; CC, $28.1 \%$; $P=0.701)$. To specifically and sensitively detect the mutation for hypercholesterolaemia, Japanese American women were divided into the $\mathrm{G}$ allele and $\mathrm{CC}$ genotype, or GG genotype and $\mathrm{C}$ allele. The subjects with the $\mathrm{G}$ allele exhibited a higher prevalence of hypercholesterolaemia compared with the CC genotype by the $\chi^{2}$ test (G allele $60.0 \%$ v. CC $42.1 \% ; P=0.011)$, whereas no difference was observed for the others (GG $62.2 \% v$. C allele $54.2 \% ; P=0.061$ ).

Last, we assessed the OR of hypercholesterolaemia from logistic regression analysis in Japanese American women (Table 3). The subjects with the CC genotype had a 0.43fold decreased risk (95\% CI $0.24,0.80$ ) for hypercholesterolaemia compared with the GG genotype after adjustment for age, \%fat, smoking status and hormone replacement therapy. In addition, age and \%fat were independent risk factors for hypercholesterolaemia and the standard coefficients of them were higher than that of the SNP.

\section{Discussion}

We demonstrated that the INSIG2 upstream SNP, rs7566605, was related to the prevalence of hypercholesterolaemia in Japanese American female subjects, but not in Japanese male and female, or Japanese American male subjects. The CC genotype is an independent protective genetic factor for progressing hypercholesterolaemia on a high-fat diet, especially in female subjects.

Table 3. Association of rs7566605 genotype with hypercholesterolaemia in Japanese American women*

\begin{tabular}{lccrc}
\hline & OR & $95 \% \mathrm{Cl}$ & SC & $P$ \\
\hline rs7566605 genotype & & & & \\
GG & 1.00 & & & \\
GC & 0.77 & $0.53,1.13$ & 1.77 & 0.183 \\
CC & 0.43 & $0.24,0.80$ & 7.27 & 0.007 \\
Age (per 1 year) & 1.02 & $1.01,1.04$ & 11.28 & 0.001 \\
\%Fat (per 1\%) & 1.04 & $1.01,1.07$ & 9.45 & 0.002 \\
Smoking status† & 0.97 & $0.74,1.27$ & 0.05 & 0.822 \\
HRT (yes v. no) & 0.76 & $0.51,1.15$ & 1.68 & 0.195
\end{tabular}

SC, standardised coefficient; \%Fat, percentage body fat; HRT, hormone replacement therapy.

* Multivariate logistic regression analysis was used to examine independent variables associated with hypercholesterolaemia in Japanese American women. SC and $P$ values were determined by multiple logistic analyses including age, \%fat, smoking status and HRT

† Smoking status: $0=$ none; $1=$ current or past.
We examined the hypothesis that the rs7566605 SNP influences the prevalence of dyslipidaemia including hypercholesterolaemia or hypertriacylglycerolaemia, and this is the first report to prove the associations. The relationship between insig-2 and cholesterol metabolism has been reported by experimental investigations in which insig- 2 regulated the degradation of HMG-CoA reductase ${ }^{(2,3)}$. Furthermore, the complex of sterol regulatory element-binding proteins (SREBP) and SREBP cleavage-activating protein is essential for transport from the endoplasmic reticulum to the Golgi when SREBP processing occurs and genes encoding enzymes required for synthesis of cholesterol are activated in the $\operatorname{Golgi}^{(13,14)}$. Under conditions of sterol excess, insig-2, which plays an important role in regulating cholesterol metabolism, prevents such movement through binding to the complex, thereby blocking the synthesis of cholesterol in vitro ${ }^{(15)}$. Together with these experimental data, the INSIG2 upstream SNP may regulate the expression of insig-2, consequently influencing the prevalence of hypercholesterolaemia. On the other hand, insig-2-overexpressing mice exhibited decreased plasma TAG levels ${ }^{(4)}$, while the significant relationship between the SNP and prevalence of hypertriacylglycerolaemia was not obtained.

The rs7566605 CC genotype may be a protective gene factor against hypercholesterolaemia only in Japanese Americans, but not in Japanese. In considering the underling mechanisms, two possibilities are suggested. First, under conditions of sterol excess, insig-2 is known to be overexpressed $^{(15)}$. In Japanese Americans who have a habit of increased ingestion of lipids, insig-2 might be overexpressed with the CC genotype, thereby suppressing the development of hypercholesterolaemia in subjects with the genotype. Second, it has been demonstrated that insig-2 mRNA is downregulated by insulin in livers of experimental animals ${ }^{(16)}$. In other words, insulin is a factor that determines the amount of insig-2 expression. Japanese Americans presented with higher serum insulin levels compared with the Japanese in this and a previous study (Tables 1 and 2$)^{(17)}$. Therefore, the location at which the SNP is found might be important for the regulation of insig-2 due to insulin, and, thus, insig- 2 with the CC genotype could not be down-regulated by high serum levels of insulin. However, this should be confirmed by functional analyses of the SNP and further in vivo and vitro investigations.

The Japanese American women with the CC genotype had an independent risk reduction of 0.43 -fold for developing hypercholesterolaemia, compared with the GG genotype. However, the influence of genetic factors was not more significant than environmental factors, such as \%fat, on the result of the logistic regression analysis. Therefore, even if subjects have the $\mathrm{CC}$ genotype, it is important to take care of their meal contents, especially fat content.

In male subjects, unlike female subjects, the CC genotype of the rs7566605 SNP was unrelated to the prevalence of dyslipidaemia including hypercholesterolaemia. The effect of the SNP on the advancement of dyslipidaemia on a high-fat diet might be different between men and women. Another possibility exists that, in male rather than female subjects, more environmental factors, such as mental stress, alcohol consumption, etc. independent of high-fat diet might induce the prevalence of dyslipidaemia. We actually reported that a family history of diabetes, which is considered as a basic 
genetic factor, is important for the development of diabetes, only in female Japanese Americans ${ }^{(18)}$.

We could not provide any support for the results reported by Herbert et al. that the rs 7566605 SNP is associated with obesity, as assessed by BMI in five out of six cohorts ${ }^{(5)}$. Furthermore, we suggest that other parameters related to obesity, such as waist girth and \%fat, were also not influenced by the SNP. However, they and other researchers suggested that the effect of the SNP on BMI may be a significant risk factor for more obese subjects, classified as BMI $>30 \mathrm{~kg} / \mathrm{m}^{2}$, but not for lean subjects as in the current population $^{(5,6,19,20)}$.

Some limitations of the present study should be taken into consideration when interpreting the present results. First, about one-quarter of Japanese American female subjects received hormone replacement therapy. The possibility exists that the lipid profile might be influenced by the therapy. In addition, since there were differences of the age between Japanese American and Japanese women, the present results might be influenced by the age of the subjects. However, even after adjustment for hormone replacement therapy and age, the significant relationship between the SNP and prevalence of hypercholesterolaemia remained. Second, we investigated the association between the SNP and hypercholesterolaemia, by assessing the prevalence, but not actual survey values of total cholesterol, TAG, or HDL-cholesterol. Since the present study included many subjects who were treated for dyslipidaemia, we could not exclude them. Third, details of antidyslipidaemic medication were undetermined in the Japanese population. However, even if the antidyslipidaemic medication was all statins or fibrate in subjects with GG or GC, statistical significance was not obtained among the genotypes in the prevalence of hypercholesterolaemia or hypertriacylglycerolaemia. Thus, we could accept the results that there were no associations between the SNP and prevalence of hypercholesterolaemia or hypertriacylglycerolaemia in Japanese men and women.

In summary, it is suggested that the CC genotype of the INSIG2 upstream SNP, rs7566605, is a protective genetic factor against hypercholesterolaemia, when exposed to a high-fat diet. The SNP may influence the expression of insig-2, consequently determining plasma cholesterol levels, while further clinical and experimental investigations are needed to confirm the present results and hypothesis.

\section{Acknowledgements}

There are no conflicts of interest in our reports.

\section{References}

1. Goldstein JL \& Brown MS (1990) Regulation of the mevalonate pathway. Nature 343, 425-430.

2. Sever N, Yang T, Brown MS, Goldstein JL \& DeBose-Boyd RA (2003) Accelerated degradation of HMG CoA reductase mediated by binding of insig- 1 to its sterol-sensing domain. Mol Cell 11, 25-33.

3. Sever N, Song BL, Yabe D, Goldstein JL, Brown MS \& DeBoseBoyd RA (2003) Insig-dependent ubiquitination and degradation of mammalian 3-hydroxy-3-methylglutaryl-CoA reductase stimulated by sterols and geranylgeraniol. J Biol Chem $\mathbf{2 7 8}$, 52479-52490.

4. Takaishi K, Duplomb L, Wang MY, Li J \& Unger RH (2004) Hepatic insig-1 or -2 overexpression reduces lipogenesis in obese Zucker diabetic fatty rats and in fasted/refed normal rats. Proc Natl Acad Sci U S A 101, 7106-7111.

5. Herbert A, Gerry NP, McQueen MB, et al. (2006) A common genetic variant is associated with adult and childhood obesity. Science 312, 279-283.

6. Lyon HN, Emilsson V, Hinney A, et al. (2007) The association of a SNP upstream of INSIG2 with body mass index is reproduced in several but not all cohorts. PLoS Genet 3, e61.

7. Nakanishi S, Okubo M, Yoneda M, Jitsuiki K, Yamane K \& Kohno N (2004) A comparison between Japanese-Americans living in Hawaii and Los Angeles and native Japanese: the impact of lifestyle westernization on diabetes mellitus. Biomed Pharmacother 58, 571-577.

8. Oki K, Yamane K, Kamei N, Nojima H \& Kohno N (2007) Circulating visfatin level is correlated with inflammation, but not with insulin resistance. Clin Endocrinol 67, 796-800.

9. Jebb SA, Cole TJ, Doman D, Murgatroyd PR \& Prentice AM (2000) Evaluation of the novel Tanita body-fat analyser to measure body composition by comparison with a four-compartment model. Br J Nutr 83, 115-122.

10. Allain CC, Poon LS, Chan CS, Richmond W \& Fu PC (1974) Enzymatic determination of total serum cholesterol. Clin Chem 20, 470-475.

11. Warnick GR, Nguyen T \& Albers AA (1985) Comparison of improved precipitation methods for quantification of high-density lipoprotein cholesterol. Clin Chem 31, 217-222.

12. Matthews DR, Hosker JP, Rudenski AS, Naylor BA, Treacher DF \& Turner RC (1985) Homeostasis model assessment: insulin resistance and $\beta$-cell function from fasting plasma glucose and insulin concentrations in man. Diabetologia 28, 412-419.

13. Nohturfft A, DeBose-Boyd RA, Scheek S, Goldstein JL \& Brown MS (1999) Sterols regulate cycling of SREBP cleavage-activating protein (SCAP) between endoplasmic reticulum and Golgi. Proc Natl Acad Sci U S A 96, 11235-11240.

14. Brown AJ, Sun L, Feramisco JD, Brown MS \& Goldstein JL (2002) Cholesterol addition to ER membranes alters conformation of SCAP, the SREBP escort protein that regulates cholesterol metabolism. Mol Cell 10, 237-245.

15. Yabe D, Brown MS \& Goldstein JL (2002) Insig-2, a second endoplasmic reticulum protein that binds SCAP and blocks export of sterol regulatory element-binding proteins. Proc Natl Acad Sci U S A 99, 12753-12758.

16. Yabe D, Komuro R, Liang G, Goldstein JL \& Brown MS (2003) Liver-specific mRNA for Insig-2 down-regulated by insulin: implications for fatty acid synthesis. Proc Natl Acad Sci U S A 100, 3155-3160.

17. Hara H, Egusa G, Yamakido M \& Kawate R (1993) Westernized food habits and concentrations of serum lipids in the Japanese. Atherosclerosis 100, 249-255.

18. Nakanishi S, Yamane K, Kamei N, Okubo M \& Kohno N (2003) Relationship between development of diabetes and family history by gender in Japanese-Americans. Diabetes Res Clin Pract 61, 109-115.

19. Hall DH, Rahman T, Avery PJ \& Keavney B (2006) INSIG-2 promoter polymorphism and obesity related phenotypes: association study in 1428 members of 248 families. BMC Med Genet 7, 83 .

20. Smith AJ, Cooper JA, Li LK \& Humphries SE (2007) INSIG2 gene polymorphism is not associated with obesity in Caucasian, Afro-Caribbean and Indian subjects. Int J Obes (Lond) 31, 1753-1755. 